\title{
Environment-dependent payoffs in finite populations
}

\author{
Weihong Xu $u^{a}$, Yanling Zhang ${ }^{a}$, Guangming Xie ${ }^{a}$ \\ ${ }^{a}$ Center for Systems and Control, State Key Laboratory for Turbulence and Complex Systems, College of Engineering, Peking University, Beijing \\ 100871, China
}

\begin{abstract}
In constant-payoff finite population games, when selection is weak and population size is large, the one-third law serves as the condition for a strategy to be advantageous. We generalize the result to the case where payoff matrices are environment-dependent and provide a more general law. In this way we model feedback from the environment and show its impact on the dynamics.
\end{abstract}

\section{Introduction}

In the 1970s, J. Maynard Smith and G.R.Price brought the concept of game into biology, and introduced the idea of evolutionary game theory to describe population dynamics (Smith et al., 1982; Smith and Price, 1973). Since then, the idea has been widely used in biology, economy and society, where how cooperation emerges among egoistic individuals is a major concern.

Nowak et al. (2004) introduced the fixation probability of a single A-individual (usually we consider a cooperator), denoted by $\rho_{A}$, which is defined as the probability that its offspring lineage invades and takes over a population of $(N-1)$ many B-individuals. If the fixation probability is bigger than that of a neutral mutant, $1 / N$, strategy $\mathrm{A}$ is deemed advantageous. Under weak selection, computation has shown that $\rho_{A}>1 / N$ for large $N$ if

$$
a+2 b>c+2 d
$$

where $a, b, c$ and $d$ are the payoffs of A versus A, A versus B, B versus A and B versus B, or denoted with a payoff matrix

$$
\begin{gathered}
A \\
B \\
B
\end{gathered}\left(\begin{array}{ll}
a & c \\
b & d
\end{array}\right)
$$

This condition is equivalent of requiring the fitness of an A-player to be higher than that of a B-player when the frequency of A is 1/3, and was dubbed the one-third law. Ohtsuki et al. (2007) provided an intuition of the law.

In most game-theoretical studies, the payoff matrices are constant, but some recent studies have been trying to model feedback from the environment by varying the entries of the payoff matrices to the state of the system (Tomochi and Kono, 2002; Lee et al., 2011).

In our paper, we treat finite population games with environment-dependent payoffs as a generalization of the traditional model. In the general case, we provide the condition for a strategy to be advantageous, of which the onethird law is a specialization. We then apply our findings to the prisoner's dilemma, the snowdrift game (also called hawk-dove game or chicken game), and cases in between, to show what effect feedback from the environment brings to the evolution.

\section{Model and the condition for a strategy to be advantageous}

We discuss the situation where the payoff matrix

$$
\begin{gathered}
A \\
A \\
B
\end{gathered}\left(\begin{array}{cc}
a(j) & c(j) \\
b(j) & d(j)
\end{array}\right)
$$


varies with the number of A-players $j$, representing feedback from the environment. For simplicity, $a(j), b(j), c(j)$ and $d(j)$ are assumed linear functions of $j$, i.e., $a(j)=a_{0}+a_{1} j, b(j)=b_{0}+b_{1} j, c(j)=c_{0}+c_{1} j$, and $d(j)=d_{0}+d_{1} j$.

The model studied by Nowak et al. and Ohtsuki et al., where $a_{1}=b_{1}=c_{1}=d_{1}=0$ (Nowak et al., 2004; Ohtsuki et al., 2007), can be considered a special case. In Ohtsuki et al. (2007), it was shown that in the limit of large $N$, one-third of the opponents that one meets (in interactions leading to state changes) until either extinction or fixation are A-players and two-thirds are B-players, and condition (1) is the exactly the same as the condition that the average payoff of A-players along an invasion-path is higher than that of B-players. In our generalized situation, though one still meets B-players twice as often as A-players, the average payoffs along an invasion-path cannot be calculated as easily. Instead, we should take into consideration that $a, b, c$ and $d$ vary with $j$. From

$$
\begin{array}{r}
\frac{1}{N-1} \sum_{j=1}^{N-1} \bar{\sigma}_{1 j}\left(\begin{array}{cc}
\left.(A \rightarrow A)\right|_{j} a(j) & \left.(A \rightarrow B)\right|_{j} b(j) \\
\left.(B \rightarrow A)\right|_{j} c(j) & \left.(B \rightarrow B)\right|_{j} d(j)
\end{array}\right) \\
=\frac{1}{N-1} \sum_{j=1}^{N-1} \frac{2(N-j)}{N}\left(\begin{array}{cc}
(j-1)\left(a_{0}+a_{1} j\right) & (N-j)\left(b_{0}+b_{1} j\right) \\
j\left(c_{0}+c_{1} j\right) & (N-j-1)\left(d_{0}+d_{1} j\right)
\end{array}\right),
\end{array}
$$

where $\bar{\sigma}_{1 j}$ is the relative frequency that state $j$ is visited (Ohtsuki et al., 2007), the average payoff of A-players along an invasion-path is

$$
\begin{array}{r}
\frac{1}{N-1} \sum_{j=1}^{N-1} \frac{2(N-j)}{N(N-1)}\left[(j-1)\left(a_{0}+a_{1} j\right)+(N-j)\left(b_{0}+b_{1} j\right)\right] \\
=\frac{1}{6(N-1)}\left[(\underbrace{a_{1}+b_{1}}_{A_{2}}) N^{2}+(\underbrace{2 a_{0}-a_{1}+4 b_{0}+b_{1}}_{A_{1}}) N+(\underbrace{-4 a_{0}-2 a_{1}-2 b_{0}}_{A_{0}})\right],
\end{array}
$$

and that of B-players is

$$
\begin{array}{r}
\frac{1}{N-1} \sum_{j=1}^{N-1} \frac{2(N-j)}{N(N-1)}\left[j\left(c_{0}+c_{1} j\right)+(N-j-1)\left(d_{0}+d_{1} j\right)\right] \\
=\frac{1}{6(N-1)}\left[(\underbrace{c_{1}+d_{1}}_{B_{2}}) N^{2}+(\underbrace{2 c_{0}+c_{1}+4 d_{0}-d_{1}}_{B_{1}}) N+(\underbrace{2 c_{0}-8 d_{0}-2 d_{1}}_{B_{0}})\right] .
\end{array}
$$

Therefore, for large $N$, the condition for strategy A to be advantageous is

$$
A_{2}>B_{2}
$$

or

$$
\left\{\begin{array}{l}
A_{2}=B_{2} \\
A_{1}>B_{1}
\end{array},\right.
$$

or

$$
\left\{\begin{array}{l}
A_{2}=B_{2} \\
A_{1}=B_{1} \\
A_{0}>B_{0}
\end{array} .\right.
$$

Here $a_{1}, b_{1}, c_{1}$ and $d_{1}$ are the main factors because $N$ is so large that on the path of invasion, for a significant amount of time, $j$ is large enough that $a_{1} j \gg a_{0}$, similar for $b(j), c(j)$ and $d(j)$. When $a_{1}=b_{1}=c_{1}=d_{1}=0$, we obtain the one-third law as a special case.

The above is verified with direct calculation of $\rho_{A}$.

$$
\rho_{A}=1 /\left(1+\sum_{k=1}^{N-1} \prod_{j=1}^{k} \frac{g_{i}}{f_{i}}\right)
$$


(Nowak et al, 2004), where $f_{i}$ and $g_{i}$ are fitness of strategies A and B, respectively:

$$
f_{i}=1-w+w[a(j)(j-1)+b(j)(N-j)] /(N-1)
$$

and

$$
g_{i}=1-w+w[c(j) j+d(j)(N-j-1)] /(N-1),
$$

with $w(w \ll 1)$ being the intensity of selection. Under weak selection, calculations yield

$$
\rho_{A}=\frac{1}{N}+\frac{\beta}{12 N}\left[\left(A_{2}-B_{2}\right) N^{2}+\left(A_{1}-B_{1}\right) N+\left(A_{0}-B_{0}\right)\right]
$$

Thus for large $N$, the condition for $\rho_{A}>1 / N$ is $(7)$ or (8) or (9).

The payoff matrix of both the prisoner's dilemma and the snowdrift game can be denoted with two parameters , the benefit $b$ and the cost $c(b>c)$ (Fu et al., 2010). As an example, we study the case where $b$ is a monotonically increasing linear function of the number of cooperators $j$ in a group of fixed size $N$, i.e., $b(j)=p+q j(p>1$ and $q>0$ ). For simplicity, we set $c=1$. This arrangement can be understood that in a society where more individuals cooperate, cooperation produces greater benefit, yet there is also a stronger temptation to defect.

The prisoner's dilemma has the payoff matrix

$$
\begin{gathered}
C \\
C \\
D
\end{gathered}\left(\begin{array}{cc}
b(j)-1 & -1 \\
b(j) & 0
\end{array}\right) .
$$

Thus, $A_{2}=a_{1}+b_{1}=q=c_{1}+d_{1}=B_{2}$, and we need to compare $A_{1}$ and $B_{1}$. Here $A_{1}=2 a_{0}-a_{1}+4 b_{0}+b_{1}=$ $2(p-1)-q+4(-1)+0=2 p-q-6$, and $B_{1}=2 c_{0}+c_{1}+4 d_{0}-d_{1}=2 p+q+4 \times 0-0=2 p+q$. Therefore $A_{1}<B_{1}$, which suggests cooperation is disadvantageous (i.e., the fixation probability of a single cooperator $\rho_{C}$ is smaller than $1 / N)$.

In contrast, the snowdrift game has the payoff matrix

$$
\begin{gathered}
C \\
C \\
D
\end{gathered}\left(\begin{array}{cc}
b(j)-1 / 2 & b(j)-1 \\
b(j) & 0
\end{array}\right)
$$

Thus, $A_{2}=a_{1}+b_{1}=2 q$, and $B_{2}=c_{1}+d_{1}=q$. Therefore $A_{2}>B_{2}$, suggesting that cooperation is advantageous (i.e., $\left.\rho_{C}>1 / N\right)$.

When $b$ is a constant, the one-third law yields $b-3>b$ for the prisoner's dilemma, which is impossible, and $3 b-3 / 2>b$ for the snowdrift game, which is, however, satisfied for all $b$ (note that we already require $b>1$ ). Therefore, cooperation is advantageous in the snowdrift game but not in the prisoner's dilemma, which is still the case, as we have shown, when $b$ is a monotonically increasing linear function of $j$.

While this may not be very interesting so far, we further consider the cases in between. There are situations not so bad as the prisoner's dilemma and at the same time not as good as the snowdrift game. We introduce a parameter $x$ $(0<x<1)$, which is the closeness of the payoff matrix to that of the prisoner's dilemma, to represent those. And we use a linear combination of the payoff matrices of the prisoner's dilemma and the snowdrift game to form our payoff matrix:

$$
\begin{aligned}
& C \quad D \\
& { }_{D}^{C}\left(\begin{array}{cc}
x[b(j)-1]+(1-x)[b(j)-1 / 2] & -x+(1-x)[b(j)-1] \\
x b(j)+(1-x) b(j) & 0
\end{array}\right) .
\end{aligned}
$$

$x$ and $1-x$ may also be understood as the probabilities that the prisoner's dilemma and the snowdrift game are being played, respectively, and our game is a combination of the two. $A_{2}>B_{2}$ ( i.e., $a_{1}+b_{1}>c_{1}+d_{1}$ ) yields $q x+2 q(1-x)>q x+q(1-x)$, i.e., $q(1-x)>0$, which is satisfied automatically. However, if $b$ is constant, the one-third law (1) requires $(b-3) x+(3 b-3 / 2)(1-x)>b x+b(1-x)$, i.e., $b \geq-3 / 4+3 / 2(1-x)$. This is satisfied only when $b$ is big enough. In particular, when $x$ approximates 1 , this will require $b$ to approximate infinity. Therefore, we arrive at an interesting conclusion: this increase of $b$ with $j$ is helpful in establishing the dominance of cooperation in cases between the prisoner's dilemma and the snowdrift game (or cases that are combinations of the two). When $b$ is a monotonically increasing function of $j$, a minimum portion of the snowdrift game is needed to transform the prisoner's dilemma into a game where cooperation is advantageous. 


\section{Conclusion}

We derived the condition for strategy A to be advantageous in finite population games, when selection is weak, population size is large, and the payoff matrix varies with the number of A-players. When the payoff matrix is constant, the condition becomes the one-third law given by Nowak et al. (2004).

The condition was then applied to the study of the prisoner's dilemma, the snowdrift game, and their combinations, whose payoff matrices can all be denoted with two parameters, the benefit $b$ and the cost $c$ (Fu et al., 2010). While an increase of $b$ with the number of cooperators has little significance in reshaping the prisoner's dilemma, where cooperation is disadvantageous, or the snowdrift game, where cooperation is advantageous, it plays a rather important role in cases that are combinations of the two.

\section{References}

Nowak, M.A., Sasaki, A., Taylor, C., Fudenberg, D., 2004. Emergence of cooperation and evolutionary stability in finite populations. Nature 428, $646-650$.

Smith, J.M., 1982. Evolution and the theory of games. J. Math. Biol. 54, 721-744.

Smith, J.M., Price, G.R., 1973. The logic of animal conflict. Nature 246, 15-18.

Ohtsuki, H., Bordalo, P., Nowak, M.A., 2007. The one-third law of evolutionary dynamics. J. Theor. Biol. 249(2), 289-295.

Tomochi, M., Kono, M., 2002. Spatial prisoner's dilemma games with dynamic payoff matrices. Phys. Rev. E 65, 026112.

Lee, S., Holme, P., Wu, Z., 2011. Emergent hierarchial structures in multiadaptive games. Phys. Rev. Lett. 106, 028702.

Fu, F., Nowak, M.A., Hauert, C., 2010. Invasion and expansion of cooperators in lattice populations: prisoner's dilemma vs. snowdrift games. J. Theor. Biol. 266 (3), 358-366. 